A high aspect ratio SU-8 fabrication technique for hollow microneedles for transdermal drug delivery and blood extraction

This article has been downloaded from IOPscience. Please scroll down to see the full text article.

2010 J. Micromech. Microeng. 20064006

(http://iopscience.iop.org/0960-1317/20/6/064006)

View the table of contents for this issue, or go to the journal homepage for more

Download details:

IP Address: 134.58.253.57

The article was downloaded on $02 / 06 / 2010$ at $14: 36$

Please note that terms and conditions apply. 


\title{
A high aspect ratio SU-8 fabrication technique for hollow microneedles for transdermal drug delivery and blood extraction
}

\author{
Buddhadev Paul Chaudhri ${ }^{1,2}$, Frederik Ceyssens ${ }^{1}$, Piet De Moor ${ }^{2}$, \\ Chris Van Hoof ${ }^{1,2}$ and Robert Puers ${ }^{1,2}$ \\ ${ }^{1}$ Department of Electrical Engineering, ESAT, Katholieke Universiteit Leuven, 3001 Leuven, Belgium \\ ${ }^{2}$ IMEC, Kapeldreef 75, 3001 Leuven, Belgium \\ E-mail: buddha@imec.be
}

Received 1 December 2009, in final form 9 February 2010

Published 1 June 2010

Online at stacks.iop.org/JMM/20/064006

\begin{abstract}
Protein drugs, e.g. hormonal drugs, cannot be delivered orally to a patient as they get digested in the gastro-intestinal (GI) tract. Thus, it is imperative that these kinds of drugs are delivered transdermally through the skin. To provide for real-time feedback as well as to test independently for various substances in the blood, we also need a blood sampling system. Microneedles can perform both these functions. Further, microneedles made of silicon or metal have the risk of breaking inside the skin thereby leading to complications. SU- 8 , being approved of as being biocompatible by the Food and Drug Agency (FDA) of the United States, is an attractive alternative because firstly it is a polymer material, thereby reducing the chances of breakages inside the skin, and secondly it is a negative photoresist, thereby leading to ease of fabrication. Thus, here we present very tall (around $1600 \mu \mathrm{m}$ ) SU-8 polymer-based hollow microneedles fabricated by a simple and repeatable process, which are a very good candidate for transdermal drug delivery as well as blood extraction. The paper elaborates on the details that allow the fabrication of such extreme aspect ratios $(>100)$.
\end{abstract}

\section{Introduction}

Usually most drugs are taken orally in the form of pills or tablets. This is acceptable for the conventional type of drugs, though it reduces their efficacy due to the first pass mechanism through the stomach and the GI tract. The second conventional method by which the same drugs can be administered is by injections, which is painful and requires medical expertise. However, protein-based drugs, such as hormonal drugs, cannot be subjected to the first pass mechanism, as they would be digested in the process, thereby leading to complete loss of efficacy. These are typically large-molecule drugs, so they also find great resistance in transport through the skin layer in other drug delivery methods such as iontophoresis, electroporation or other topical applications to the skin. The most feasible and almost painless optimal mechanism for administering such drugs, without any medical expertise whatsoever, is by way of microneedles.

In fact, the reason why microneedles have gained so much prominence in research is primarily their sheer length, in the range of around 300 to $700 \mu \mathrm{m}$, which enables them to stay just short of the nerve endings in the deeper dermis layers. This length is viable for drug delivery but for blood extraction, it is required to reach into the deeper layers of the dermis to tap into the blood vessels, at around $1500 \mu \mathrm{m}$. Previously, materials such as silicon and metals [1,2] have been used predominantly for fabrication of microneedles. But silicon, though in extensive use in the microsystems industry, is fraught with many risks in the biological domain. Being fragile and prone to breakage inside the skin, in addition to its biocompatibility still unproven, are some of its disadvantages [3]. Though some metals, such as platinum, in comparison might be biocompatible, the risk of breakage still remains. It is here 


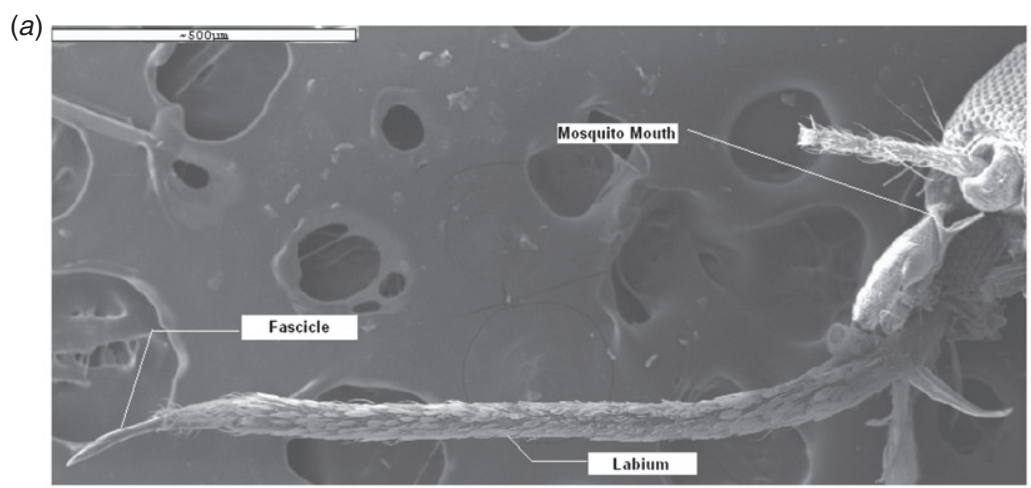

(b)

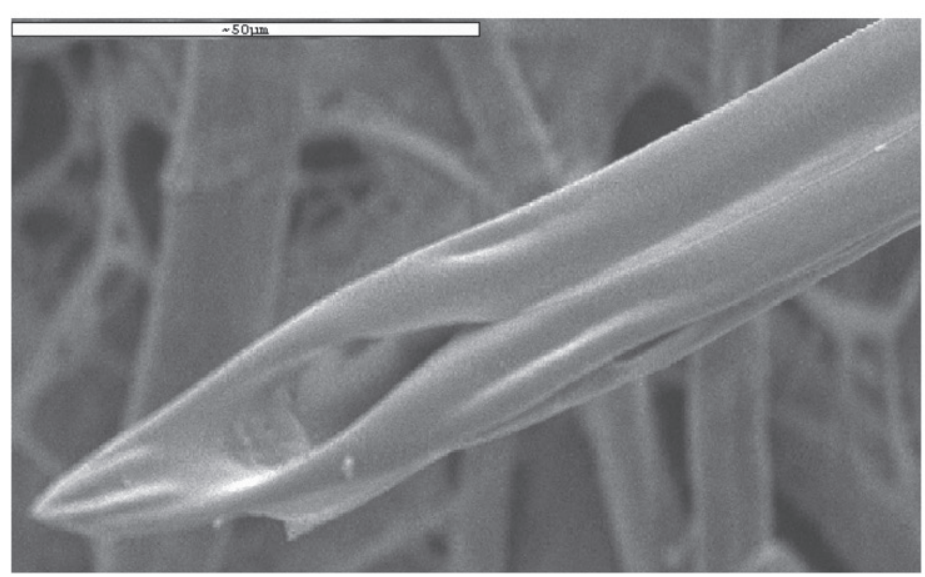

(c)

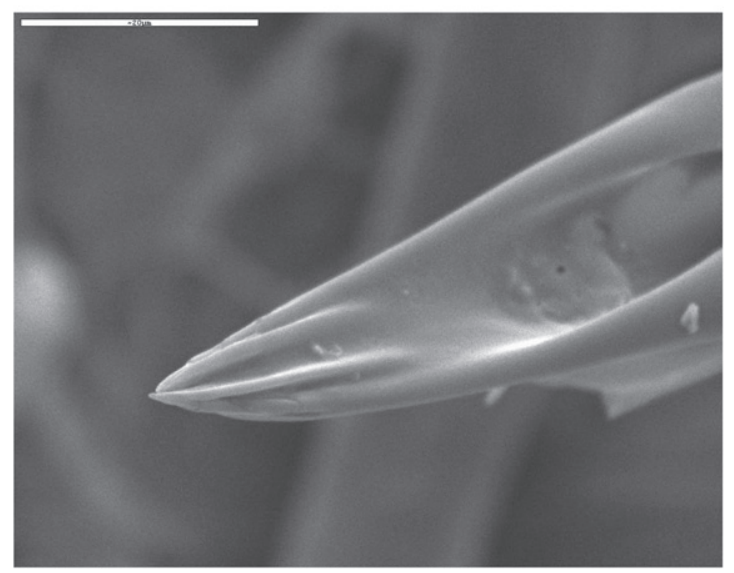

Figure 1. Anti-clockwise from top: $(a)$ a mosquito mouth and the fascicle or needle. $(b)$ A close-up view of a mosquito fascicle. Dimensions: average length: $2 \mathrm{~mm}$; average wall thickness: $4-5 \mu \mathrm{m}$; average outer diameter: $30 \mu \mathrm{m}$; average aspect ratio: 400 . (c) The reinforced tip of the fascicle (used with kind permission from [7]).

that polymer-based microneedles, which have received limited attention, score a few points, namely proven biocompatibility and improved resistance to shear-induced breakage arising from polymer visco-elasticity $[4,5]$.

Also inexpensive and mass-production techniques can be used for polymer-based production of microneedles. However, the downside with a polymer-based process is that very tall (high aspect ratio) and hollow structures are somewhat difficult to produce. In this paper, we report successfully fabricated very tall and hollow out-of-plane cylindrical structures of $1540 \mu \mathrm{m}$ height with a wall thickness of $15 \mu \mathrm{m}$ and an inner diameter of $100 \mu \mathrm{m}$. The material used is SU-8, and the process has been run successfully several times indicating good repeatability for mass production. Such high aspect ratios have been rare in the area of polymer-based hollow microneedle fabrication.

\section{Microneedle design}

\subsection{Choice of structural material}

Polymer microneedles have been fabricated from several different materials such as polylactic acid (PLA), polyglycolic acid (PGA) and their copolymers [3]. However, the advantages with SU-8 are firstly, it has a high Young's modulus of 4.02 $\mathrm{GPa}$ [6] compared to the other aforementioned materials, and secondly, it facilitates fabrication by being a negative photoresist.

\subsection{Design requirement}

2.2.1. Microneedle size. As already mentioned above, microneedles for transdermal drug delivery are not required to be longer than $700 \mu \mathrm{m}$. But for blood extraction, lengths of around $1500 \mu \mathrm{m}$ are needed. Ideally, our inspiration is derived from the mosquito fascicle and the way in which a mosquito suctions blood from the skin. The shape of the mosquito tip is that of a hypodermic syringe with a reinforced tip as can be seen from figure 1.

The final goal is to mimic the entire mosquito blood suctioning process, but the first step is to fabricate microneedles which come close to the mosquito's average aspect ratio of 400 . This is necessary as the smaller the microneedle tip diameter, the less would be the pain afflicted on a patient by way of smaller insertion force [7]. There is however a tradeoff when it comes to the fabrication of such small structures with SU-8, as we have noticed that at less than $15 \mu \mathrm{m}$ of wall thickness and at heights greater than $800 \mu \mathrm{m}$, the structures are not strong enough to even withstand the development process.

2.2.2. Microneedle mechanical characteristics. We know that the microneedle insertion force $\left(F_{i}\right)$ is directly proportional to the microneedle tip area $(A)$ [8]. If the human skin puncture toughness is given by $G_{p}$, then

$$
F_{i}=\tau G_{p} A
$$


Table 1. For SU-8 microneedle of outer radius of tip, $r_{t}=65 \mu \mathrm{m}$ and $F_{i}=0.543 \mathrm{~N}$.

\begin{tabular}{ll}
\hline Tip angle, $\alpha$ (degrees) & Failure force, $F_{b}(\mathrm{~N})$ \\
\hline 90 & 1.547 \\
54.74 & 1.050 \\
\hline
\end{tabular}

where $\tau$ has been experimentally determined to have a value of $6.25 \pm 0.6 \mathrm{~mm}^{-1}$ [8] and $G_{p}=30.1 \pm 0.6 \mathrm{~kJ} \mathrm{~m}^{-2}$ [8]. For purposes of tip area calculation, we are concerned with the interfacial area that is the effective area of contact between the needle and the skin. Since we are dealing with a hollow needle here, it is the annular surface area, $A_{a}$, that needs to be calculated at the needle tip as follows [8]:

$$
A_{a}=\pi\left(r_{t} t-\frac{t^{2}}{4}\right)
$$

where $r_{t}$ is the outer radius of the microneedle tip and $t$ is the wall thickness.

To predict the force required for the needle to fail due to buckling, Hausrath and Dittoe [11], have developed a widely used empirical modification to an analytical solution as follows:

$$
F_{b}=0.277\left(2 \pi E t^{2} \sin ^{2} \alpha\right)
$$

where $F_{b}$ is the axial load to cause buckling, $E$ is Young's modulus of the structural material and $\alpha$ is the needle tip angle.

From the basic principles of mechanical engineering, it is known that the safety factor is the ratio of $F_{b}$ to $F_{i}$ and should always be greater than unity for mechanical stability.

The inner diameter of the microneedle was designed for $100 \mu \mathrm{m}$ with $t=15 \mu \mathrm{m}$. Thus, $r_{t}=65 \mu \mathrm{m}$. $F_{i}$ can therefore be computed from (1) to be equal to $0.543 \mathrm{~N}$. Two different tip angles $\alpha$ have been considered for calculating the failure force $F_{b}$ from (3). When the needle tip is flat, $\alpha=90^{\circ}$. The second case is when $\alpha=54.74^{\circ}$. The reason for the latter value of angle is that at a later stage of this work, the microneedle tip would be sharpened by a moulding step from anisotropically etched $\mathrm{KOH}$ trenches and thus the needle tip would take the angle of the trench walls.

Thus, it can be easily seen from table 1 that in both cases the safety margin $F_{b} / F_{i}$ is much greater than unity, and therefore, the microneedles should not undergo buckling during skin penetration.

\section{Fabrication}

\subsection{Process steps}

The structural material of the microneedles fabricated is SU-8 2150 from MicroChem Inc., USA.

The process employed is fairly straightforward. Since SU-8 is basically a negative photoresist material, this property has been exploited. This enables one to circumvent the entire arduous process of isotropic etch by deep reactive ion etching (DRIE) in order to create vertical out-of-plane structures. In order to fabricate thick structures, the SU-8 is simply poured out of its bottle directly onto the $\mathrm{Si}$ wafer substrate while

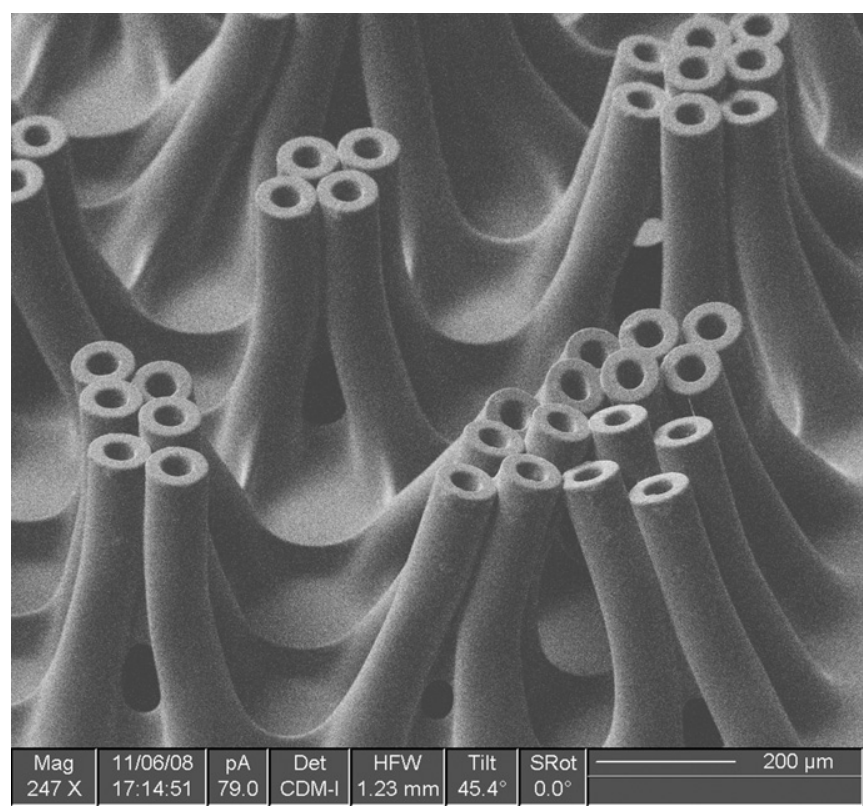

Figure 2. Result of non-uniform evaporation of solvent in the pre-exposure bake stage leading to the weak pillar base in the final structure, causing the bending and collapse.

it is lying on a precision weighing scale. The thickness is controlled by measuring the weight of the SU-8 on the wafer.

The optimum weight of SU-8 to be placed on the wafer, corresponding to a specific thickness, is obtained by trial and error. It has been observed that the conventional process flow for fabricating SU-8 structures as reported in the literature does not hold good in this case, as the SU-8 structural layer here is much thicker than is usually the case. Due to the high thickness of the layer a very typical problem is encountered, namely the large concentration gradient of the solvent from the bulk of the SU-8 material to the surface after the soft bake step. This leads to issues in the later stages in terms of basepillar strength which becomes greatly weakened as the solvent material, which is supposed to have been evaporated in the pre-exposure bake stage, did not evaporate completely from the lower layers, thus leading to this problem, as shown in figure 2.

Another severe problem that is encountered in such thick layers is the formation of a copious quantity of air bubbles. Obviously, the next step of UV exposure cannot be executed properly if these air bubbles are present. To overcome these obstacles, the pre-exposure bake was divided into two parts [9].

First, a soft bake was carried out at $95^{\circ} \mathrm{C}$ for $5 \mathrm{~h}$ on a hot plate, followed by cooling to room temperature for the next $12 \mathrm{~h}$. For this step, a stainless steel plate was utilized instead of a $\mathrm{Si}$ wafer.

Second, this entire mass of semi-dried SU-8 material was scraped out manually and collected in the form of 'dry chips'. The advantage of these dry chips is that they can be stored for a very long period of time and can be used directly instead of having to go through the time-taking first step again. These dry chips were then heated to $110^{\circ} \mathrm{C}$ in a vacuum oven. The entire process flow is as shown in figure 3 . 


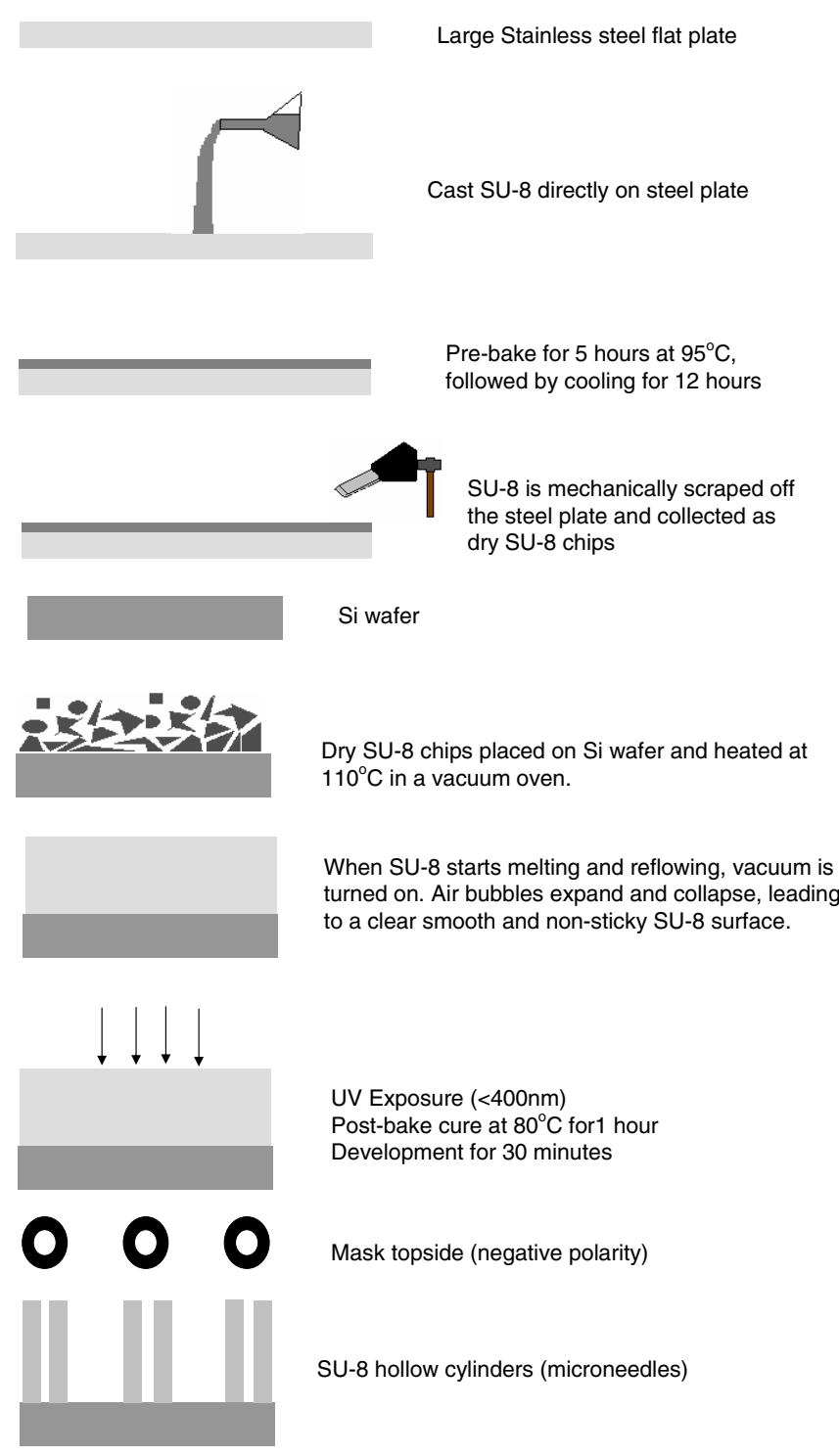

Figure 3. Detailed process flow followed for the fabrication of the high aspect ratio SU-8 microneedles.

During the vacuum oven heating step, it should be noted that at first only the heating is switched on, and not the vacuum pump. As the dry chips start melting and reflowing, the dirty looking uneven dry SU-8 surface is transformed and the SU-8 gradually spreads out smoothly over the whole wafer surface. At this juncture, if the wafer is brought out of the oven and observed closely, it would be noted that tiny bubbles have formed inside the bulk of the SU-8 layer. This is absolutely detrimental to the photolithography process which is next. Thus, when the SU-8 is observed to have fully melted and spread evenly over the full Si wafer, the vacuum pump is turned on. As the pressure inside the oven falls, it is observed that the air bubbles expand in a dramatic manner, till a certain point when it appears as if the entire wafer surface is covered with one large bubble. At the moment the air pressure reaches the lowest value of around $10^{-4} \mathrm{bar}$, suddenly the large air bubble collapses, followed by a sporadic and continuous bursting occurring all throughout the SU-8 surface till no more bubbles are to be seen. However, there is still

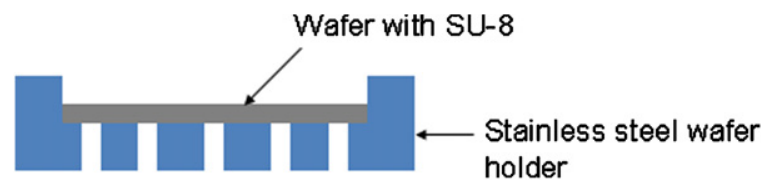

Figure 4. Stainless steel plate with through-holes and a raised rim for holding the wafer in the vacuum oven.

another issue to be grappled with. During this entire process of SU-8 melting and reflowing, followed by the air bubbles' expansion and collapse, much of the SU-8 material is lost as it tends to flow out of the wafer boundary especially when the bubbles collapse. To overcome this problem, a special round stainless steel plate with a diameter slightly greater than the wafer diameter, with through-holes and a raised boundary (the height of which should be much greater than the desired height of the needles), was constructed in order to hold the reflowed SU-8 within the wafer boundary, as shown in figures 4 and 5. So, once the bubbles have collapsed showing a clear SU-8 surface, the air pressure inside the oven is raised once again. It is to be noted that the oven temperature should still be kept at $110^{\circ} \mathrm{C}$ for reasons that shall become clear later. When the air pressure reaches normal atmospheric levels, the oven chamber is opened and the wafer holder containing the wafer is brought out. It will now be observed that due to the reflow and the sporadic bursting of the air bubbles, the SU-8 material has spread everywhere, and thus the wafer in all probability would now be stuck to the bottom of the stainless steel wafer holder. But since the temperature was maintained at $110{ }^{\circ} \mathrm{C}$, when the wafer is brought out, the SU-8 is still in a semi-molten state. Acetone is poured via the through-holes at the bottom of the wafer holder to dissolve the SU-8 sticking to the bottom of the wafer, and a pair of tweezers is used to gently take the wafer off the stainless steel surface. This step is a very critical one and must be done as quickly as possible, because the more time consumed to take the wafer off the stainless steel surface, the more difficult it becomes to do so as the SU-8 cools down very rapidly preventing further removal. The holes at the bottom of the stainless steel surface serve to allow acetone to reach the bottom surface of the wafer to allow the detachment of the SU-8. This step would have been much simpler had the wafer been allowed to cool down substantially, but the fact that it is to be done immediately after taking out of the oven, makes this step the most challenging yet apparently very trivial.

Once this step has been completed, the SU-8 is allowed to cool down to room temperature. The surface texture is now smooth and non-sticky with no air bubbles. The SU-8 is then subjected to UV exposure. To ensure even exposure throughout the layer, care should taken to ensure that wavelengths below $400 \mathrm{~nm}$ are filtered out of the UV spectrum before exposure [10]. An exposure energy of $12 \mathrm{~J} \mathrm{~cm}^{-2}$ is used.

Next, a post-exposure bake step is carried out for the exposed SU-8, in an oven, at $80^{\circ} \mathrm{C}$, for $1 \mathrm{~h}$. After baking, temperature is ramped down to room temperature over a period of $3 \mathrm{~h}$.

This is followed by a development step in an ultrasonic bath for $30 \mathrm{~min}$. 


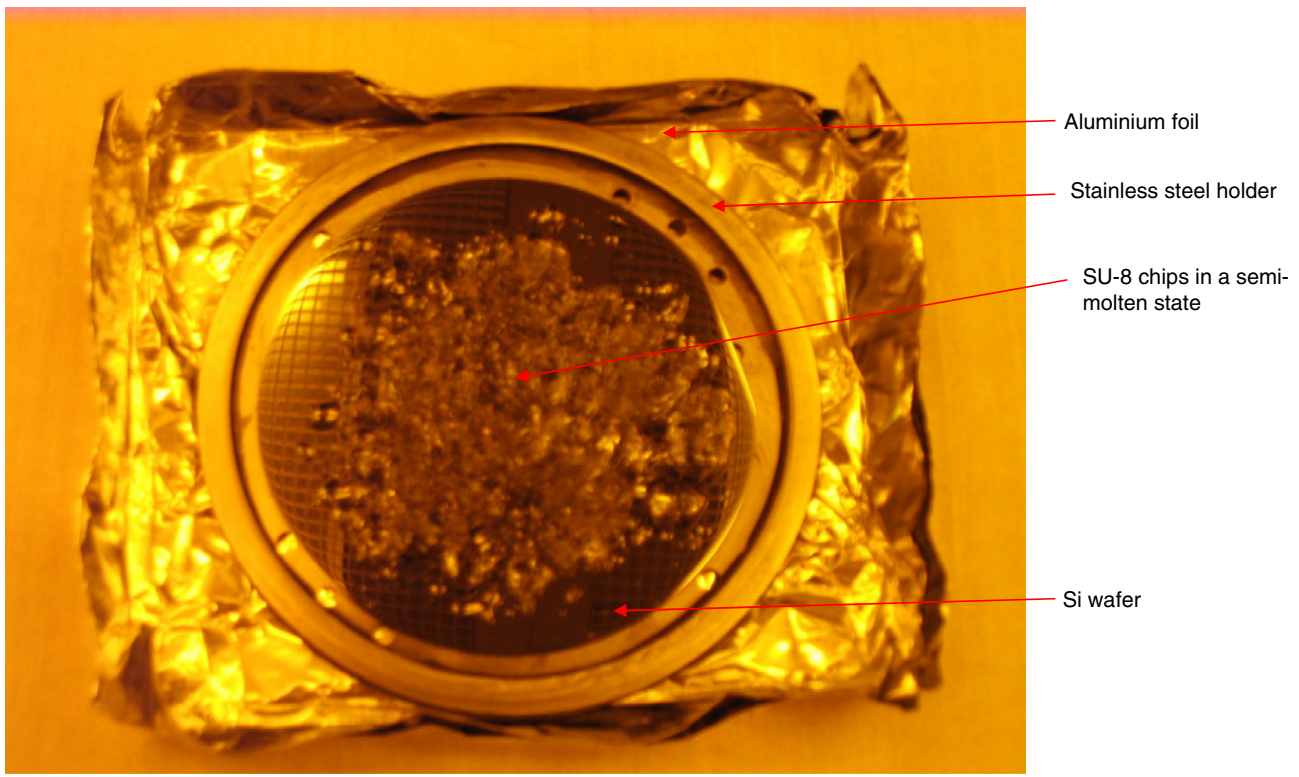

Figure 5. Semi-molten SU-8 chips in the process of being melted inside the oven for the second soft-bake step.

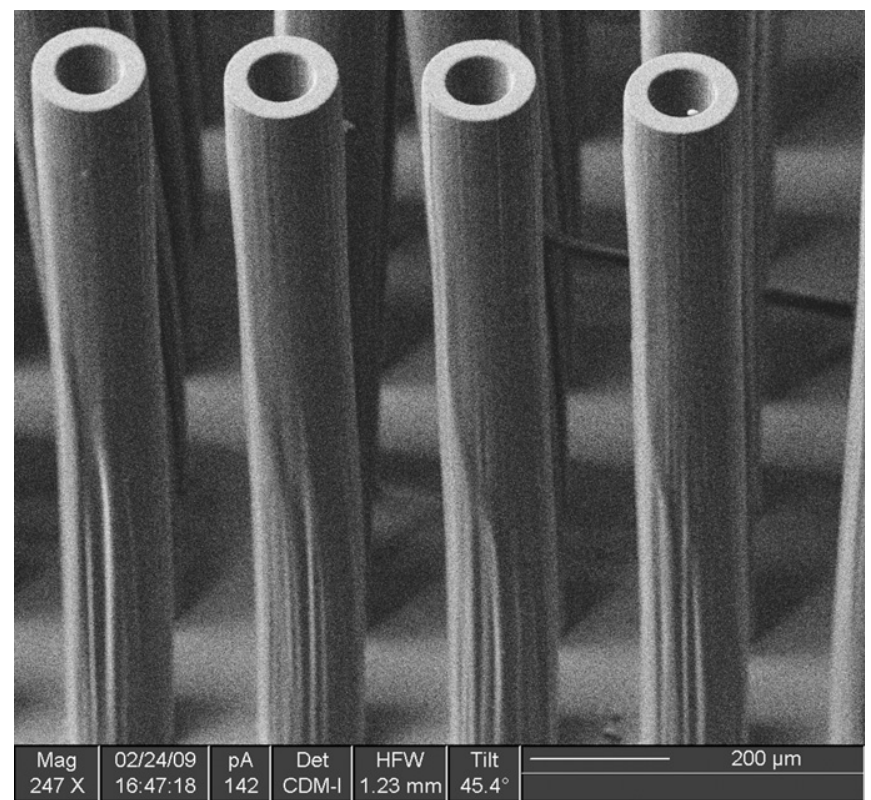

Figure 6. SU-8 hollow microneedles. Height $1540 \mu \mathrm{m}$; wall thickness $15 \mu \mathrm{m}$; inner diameter $100 \mu \mathrm{m}$.

\subsection{Fabrication results}

As can be seen from figure 6, the measured dimensions of the fabricated microneedles were an inner diameter of $100 \mu \mathrm{m}$ and a wall thickness of $15 \mu \mathrm{m}$. With a measured height of $1540 \mu \mathrm{m}$, an aspect ratio of around 103 has been achieved. Further, the walls of the microneedles are quite straight and the edges are sharp and well defined. Different cross-sectional shapes of microneedles were also fabricated as high aspect ratio structures as shown in figures 7 and 8. The latter shows triangular cross-sectioned ones with circular hollows as the lumen.

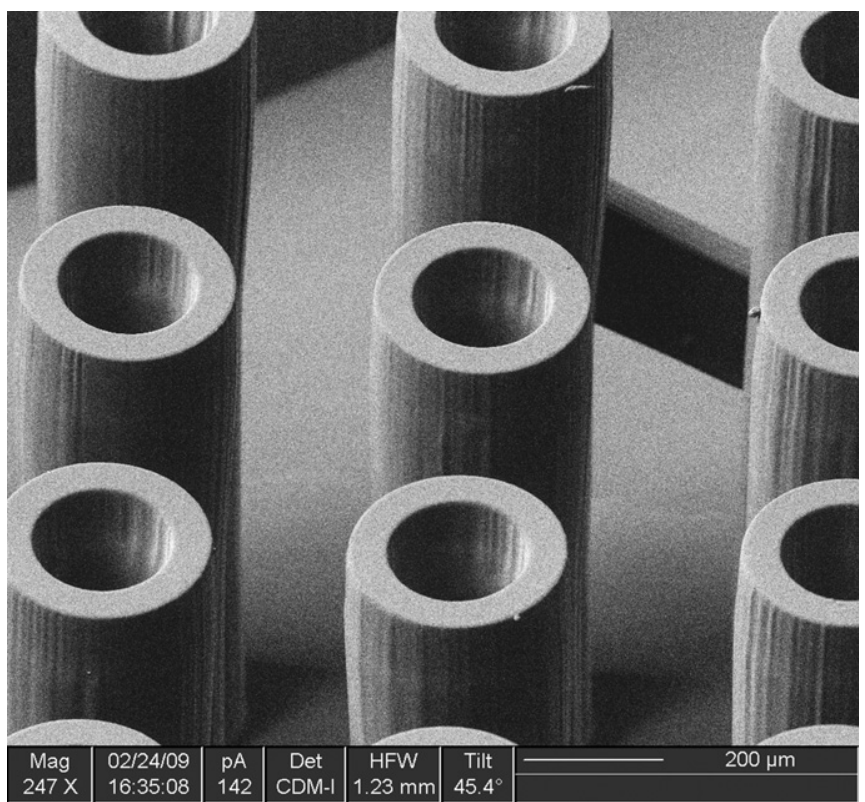

Figure 7. Closer view of another part of the microneedle array; internal needle diameter $200 \mu \mathrm{m}$.

\subsection{Testing longitudinal cross-section of fabricated microneedles}

To investigate whether the fabricated microneedles are hollow right to the bottom, a small section of the wafer substrate holding the needles was removed by dicing and moulded in epoxy, keeping the orientation of the needles such that the longitudinal axis of the needles is parallel to the grinding plane. The epoxy mould was ground manually and checked intermittently under the microscope. It has been observed that the microneedles' bore was open right up to the bottom as can be seen from figure 9. The presence of air bubbles in some of the needles, and epoxy in the rest proves that it is hollow inside. 


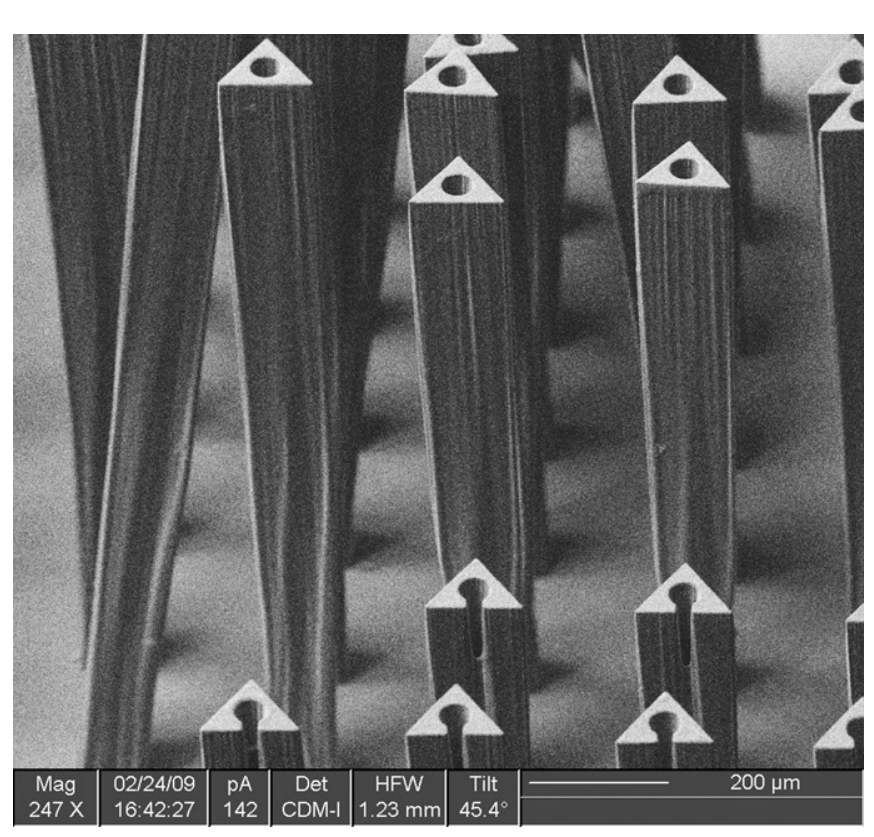

Figure 8. Triangular cross-section microneedles with a circular hollow bore (dimensions-base: $70 \mu \mathrm{m}$; circular lumen diameter = $48 \mu \mathrm{m})$. The second type of structure lower down in the figure is one in which the hollow of the needle has been intentionally kept open by mask design, to allow for a clear view of the longitudinal axis. The substrate surface is clear indicating a successful SU-8 development step.

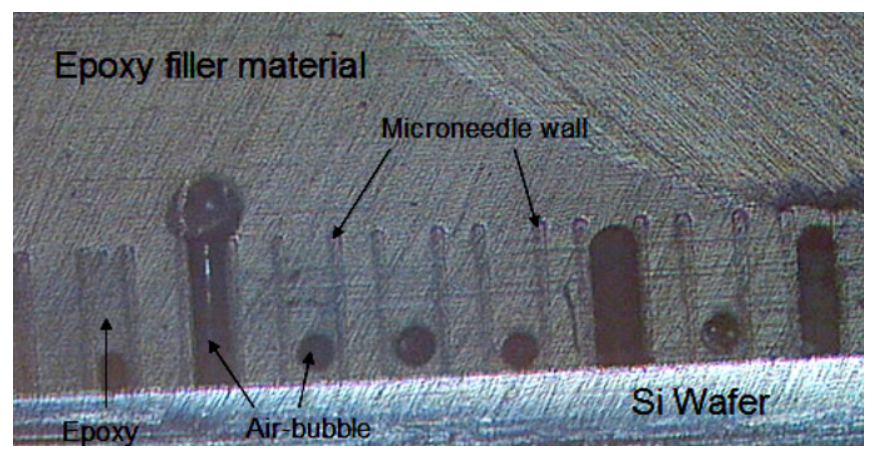

Figure 9. Cross-sectional view of fabricated SU-8 microneedles moulded in epoxy, indicating that the needles are hollow right to the bottom.

\section{Conclusions and future work}

High aspect ratio, polymer hollow microneedles were fabricated out-of-plane in a simple manner using a relatively inexpensive polymer material, namely SU-8. This is a significant step forward towards practical and yet low-cost transdermal delivery and sampling systems. Further, it was also clear from figure 9 that the bores of the microneedles are open right to the bottom. Though the wall thickness of $15 \mu \mathrm{m}$ is sufficient for piercing the skin with adequate insertion force, work is on-going to sharpen the needle tips, as smaller needle tip area leads to a smaller insertion force requirement, which again means less pain inflicted on the skin. Towards this end, we are working on moulding the microneedle tips on $\mathrm{KOH}$-etched grooves in a $\langle 100\rangle \mathrm{Si}$ wafer. Currently, we have successfully moulded SU-8 on these anisotropically etched pyramidal trenches. Using this technique to sharpen the needle tips is the next step. The testing of the mechanical strength of such long microneedles is in progress.

\section{Acknowledgments}

This work would not have been possible without the use of the ESAT (Department of Electrical Engineering, KU Leuven, Belgium) clean-room facilities. This project is carried out under the auspices of IMEC (Interuniversity Microelectronics Centre, Leuven, Belgium) doctoral grant, and the authors would like to express their gratitude to all the people concerned for their support.

\section{References}

[1] Zahn J D et al 2000 Microfabricated polysilicon microneedles for minimally invasive biomedical devices Biomed. Microdevices 2 295-303

[2] Stoeber B et al 2000 Two-dimensional arrays out of plane needles ASME Int. Mechanical Engineering Congress and Exposition, MEMS (Orlando, FL)

[3] Park J-H et al 2005 Biodegradable polymer microneedles: fabrication, mechanics and transdermal drug delivery J. Control. Release 104 51-66

[4] Ratner B D et al 1996 Biomaterials Science: An Introduction to Materials in Medicine (New York: Academic)

[5] Wouters K and Puers R 2009 Determing the Young's modulus and creep effects in three different photo-definable epoxies for MEMS applications Sensors Actuators A, doi:10.1016/j.sna.2009.03.021

[6] Roch I 2003 Fabrication and characterization of an SU-8 gripper actuated by a shape memory alloy thin film J. Micromech. Microeng. 13330

[7] Swaminathan V S 2006 Mechanics of a mosquito bite MS Thesis Department of Mechanical Engineering, North Caroline State University, USA http://www.lib.ncsu.edu/theses/available/ etd-04272006-144238/unrestricted/etd.pdf

[8] Davies S P et al 2004 Insertion of microneedles into skin: measurement and prediction of insertion force and needle fracture force J. Biomech. 37 1155-63

[9] Becnel C, Desta Y and Kelly K 2005 Ultra-deep x-ray lithography of densely packed SU-8 features: I. An SU-8 casting procedure to obtain uniform solvent content with accompanying experimental results $J$. Micromech. Microeng. 15 1242-8

[10] Yang R and Wang W 2005 A numerical and experimental study on gap compensation and wavelength selection in UV-lithography of ultra-high aspect ratio SU-8 microstructures Sensors Actuators B 110 279-88

[11] Hausrath A H and Dittoe A F 1962 Development of design strength levels for the elasticity stability of monocoque cnes under axial compression. Collected Papers on Instability for Shell Structures, National Aeronautics and Space Administration Technical Note D-1510 pp 45-6 